Conclusion Respiratory and gastrointestinal diseases and accidents were predominant causes of hospitalisation of children under 2 years of age. The results demonstrate that prevention activities could be essential strategies in order to reduce proportions of hospitalisation among children under 2 years of age in Brazil at least by half. Besides, this study suggests that such investment in health promotion could improve the health profiles of children in developing countries.

\section{P1-201 USING EPIDEMIOLOGY DATA FOR EVALUATING OF ECONOMIC BURDEN OF CARDIOVASCULAR DISEASES IN RUSSIAN FEDERATION}

doi:10.1136/jech.2011.142976d.94

\author{
A Kontsevaya, ${ }^{*}$ A Kalinina, I Koltunov. National research center for preventive \\ medicine, Moscow, Russia
}

Purpose To study the economic burden of cardiovascular diseases (CVD) in Russian Federation in 2006-2009.

Methods The economic burden was calculated by the cost of illness method. The calculations included direct costs of healthcare system and indirect costs, associated with premature death in working age and disability. We used official statistics of healthcare resources utilisation, associated with CVD, the results of epidemiological surveys of CVD, mortality statistics in Russia in 2006-2009.

Results The total economic burden of CVD increased from 20.6 billions of euro in 2006 till 26.6 billion of euro in 2009 what was equal to $3.1 \%-2.8 \%$ of GDP of Russian Federation. The increasing of the burden was mainly caused by the price increasing and in some degree by the increasing of PCI in CHD patients in recent years. Direct costs accounted $21.3 \%$ of total cost of CVD (5.7 billions of euro), indirect costs-78.7\% (20.9 billions of euro) in 2009. Indirect costs mainly consisted of the GDP losses because of premature death of working age men. CHD represented $37.8 \%$ and cerebrovascular diseases $17.1 \%$ of overall CVD costs. In-hospital care represented $47.5 \%$ of direct costs, out hospital visits-21.8\%, medication $-20.7 \%, \mathrm{PCI}-4.1 \%$ and emergency care $4.1 \%$ of direct costs in 2009 , respectively. CHD represented $45.3 \%$ of direct cost, because of large duration of hospitalisation and PCI costs.

Conclusion CVD is a big public health challenge in Russia. The results of economic burden assessment should help policy makers evaluate policy impact and prioritise expenditures.

\section{P1-202 MONITORING OF SOCIO-DEMOGRAPHIC DIFFERENCES IN HEALTH BEHAVIOURS IN LITHUANIA}

doi:10.1136/jech.2011.142976d.95

V Kriaucioniene, ${ }^{*}$ J Klumbiene, J Petkeviciene. Institute for Biomedical Research, Medical Academy, Lithuanian University of Health Sciences, Kaunas, Lithuania

Introduction Monitoring of heath behaviour on a national level is an important vehicle for health promotion and disease prevention, planning and evaluation. The aim of the study was to analyse the time trends of socio-demographic differences in behaviours of population.

Methods From 1994 to 2010 nine health behaviour surveys have been carried out in Lithuania. For every survey random sample of 3000 Lithuanians aged 20-64 was taken from the National Population Register. The response rates varied from $54 \%$ to $74 \%$. The study material was collected by mailed questionnaires covering socio-demographic characteristics and health behaviours factors.

Results Over the last 16 years the prevalence of daily smoking has decreased significantly among men (from $43.8 \%$ in 1994 to $34.2 \%$ in 2010 ) but it increased among women (from $6.8 \%$ to $15 \%$ respectively). Daily smoking and passive smoking at work has remained more prevalent among less educated persons than among highly educated persons. Bear consumption increased in both genders in all educational groups. The frequency of strong alcohol drinking rose among women in all educational groups, while among men it remained stable. Since 1994 nutrition habits have changed in the direction of more healthy diet in all educational groups. Daily consumption of fresh vegetables increased from $4.5 \%$ in 1994 to $20.4 \%$ in 2010. Educational inequalities in nutrition habits have diminished, but some differences have still remained significant.

Conclusions The established sociodemographic differences in health behaviour should be taken into account in elaboration of more effective health promotion programmers in Lithuania.

\section{P1-203 SOCIAL AND GENDER DIFFERENTIALS IN CHILDHOOD MORTALITY IN RURAL NORTH INDIA}

doi:10.1136/jech.2011.142976d.96

\begin{abstract}
${ }^{1,2} \mathrm{~A}$ Krishnan, ${ }^{*} \mathrm{P}$ Dwivedi, ${ }^{1} \mathrm{~V}$ Gupta, ${ }^{1} \mathrm{C}$ Pandav, ${ }^{2} \mathrm{~N}$ Ng, ${ }^{2} \mathrm{P}$ Byass. ${ }^{1} \mathrm{All}$ India Institute of Medical Sciences, New Delhi, India; ${ }^{2}$ Umeå Centre for Global Health Research, Umea, Sweden
\end{abstract}

Introduction Achieving Millennium Development Goals on childhood mortality in developing countries requires that inequities be addressed. This study examined changing gender differentials as social and economic improvement occurred in a rural north Indian community.

Methods A dynamic cohort of children aged $<5$ years old in 10 villages under Ballabgarh HDSS were followed from $1^{\text {st }}$ January 2008 to death, age 5 or data censoring on $31^{\text {st }}$ December 2010. Data on births, mortality, caste and parental literacy are routinely collected and stored in electronic databases to which information on wealth Index was appended in 2010. Cox proportional hazards modelling was used to produce HRs for mortality in girls according to socioeconomic strata after adjustment for birth order, number of siblings and other socioeconomic variables.

Results The mortality rate for the cohort was 67.9 per 1000 live births $(B=74.4 ; G=62.4)$. This declined significantly as wealth index improved (from 102.4 to 25.4 per 1000 live births). The HR for girls declined from 1.57 (95\% CI 1.0 to 2.5) in the wealthiest to 0.67 (0.3 to 1.4 ) in the lowest tertile. There was no change in total or sex specific mortality rates according to caste or father's education. Mothers' education beyond $10^{\text {th }}$ grade resulted in sharp decline in mortality (14 per 1000 live births) but did not impact the sex differential in mortality. The sex ratio at birth was worst for population with highest wealth index and highest level of maternal educational attainment.

Conclusion Major social and gender differentials persisted in this study population with gender discrimination moving from after to before birth.

\section{P1-204 EPIDEMIOLOGY OF DISABILITY IN INDIA: OBSERVATIONS FROM A RECENT STUDY IN TWO STATES, 2009-2010}

doi:10.1136/jech.2011.142976d.97

A Kumar, ${ }^{*}$ S Husain. National JALMA Institute for Leprosy \& Other Mycobacterial Diseases (ICMR), Taj Ganj, Agra, Uttar Pradesh, India

Introduction Disability refers to functional impairment of any part of the body and may be congenital or acquired. Estimation of disability load and its causes is an important index for determining services for social justice and rehabilitation. This study aimed to estimate the community disability burden in India at the request of the Government of India and WHO. 
Materials and Methods Cluster random sampling in two states (Uttar Pradesh and Haryana) was used. A sample of 100000 people per district, with four districts per state was identified. Twenty per cent were selected from urban areas and rest from four randomly selected PHCs. In rural areas the survey was conducted with the help of ASHA workers and in Urban areas by the Urban Team. ASHA workers were provided with 2 days of comprehensive training and urban team was also trained.

Results We found that the total disability rate (TDR) was 67.3/ 10000 population-77.4/10 000 in males and 56.7/10 000 in females $(p<0.001)$. The TDR was significantly higher in urban areas than in rural areas $(83.2 / 10000$ vs $62.9 / 10000, \mathrm{p}<0.001)$. The same trend was observed at the state level. The data show that $58.2 \%$ of the total disability was due to locomotors defects only (polio, paralysis, epilepsy, congenital and trauma) followed by blindness and multiple disabilities. Further data will be available for the Congress.

\section{P1-205 A CASE CONTROL STUDY TO EVALUATE THE ASSOCIATION OF ARECA-NUT AND TOBACCO ABUSE AND ORAL PRECANCEROUS LESIONS IN NORTH INDIAN POPULATION}

doi:10.1136/jech.2011.142976d.98

S Kumar, ${ }^{*}$ D Mehrotra. King George's Medical University, Lucknow, Uttar Pradesh, India

Introduction Leukoplakia, erythroplakia, smoker's palate, and oral sub mucous fibrosis (OSMF) are potentially malignant precancerous conditions of the oral cavity which are thought to be intimately associated with the habit of chewing areca nut and tobacco either in the smoking or smokeless form.

Material and Methods A case control study was carried out in a representative North Indian population by organising oral health checkup camps. A validated questionnaire was administered to all subjects enrolled followed by their clinical examination. The suspected precancerous cases were confirmed by histopathological examination. Cases were defined on conformation of the biopsy report and matched with healthy controls. Univariate and multivariate analyses were performed and the ORs were calculated for the risk factors.

Results We found 448 cases of OSMF, 61 leukoplakia, 4 erythroplakia, 24 smokers palate and 17 oral lichen planus cases among 3136 subjects enrolled. Twelve cases confirmed as OSMF developed squamous cell carcinoma during the course of the study, however no such conversion is reported from other precancer groups till now. Strong correlation between the areca nut/tobacco products and oral precancerous lesions was observed. The OR ranged from 4.77 to 6.88 for areca nut users while for tobacco users it was 4.55-9.71, depending upon daily frequency of usage.

Conclusion The study revealed areca nut and tobacco as potential risk factors for the occurrence of oral precancer lesions.

\section{P1-206 EFFECTS OF CHILDHOOD SOCIOECONOMIC POSITION ON SUBJECTIVE HEALTH AND HEALTH BEHAVIOURS IN ADULTHOOD: HOW MUCH IS MEDIATED BY ADULT SOCIOECONOMIC POSITION?}

doi:10.1136/jech.2011.142976d.99

S Mckenzie, ${ }^{*} \mathrm{~K}$ Carter, T Blakely, V Ivory. University of Otago, Wellington, New Zealand

Introduction We examine the potential mediating role of multiple individual indicators of adult socioeconomic position (SEP) in the associations of childhood SEP with self-reported health measures and health behaviours.

Methods Data came from Wave 3 of the Survey of Family, Income and Employment. Childhood SEP was measured using parental occupation. Adult SEP was measured using education, household income, labour market activity and area deprivation. Self-rated health was assessed by a single-item measure. Psychological distress was assessed using the Kessler-10 scale. Current smoking status was derived from information on current and past cigarette use and binge drinking from information on the number of occasions of bingeing in the last 4 weeks. The associations of childhood SEP with each health outcome were determined using logistic regression, while adjusting for the mediating effects of individual adult SEP indicators.

Results Respondents from a lower childhood SEP had greater odds of being a current smoker, reporting poorer health and higher psychological distress. Two-thirds to three quarters of the association of childhood SEP with smoking (78\%), and psychological distress (66\%) and over half the association with selfrated health $(55 \%)$, were explained by educational attainment. Other adult socioeconomic indicators had much smaller mediating effects.

Conclusions The association between childhood SEP and self-rated health, psychological distress and current smoking in adulthood is largely explained through an indirect socioeconomic pathway involving education. Household income, area deprivation and labour market activity are still likely to be important as they are intermediaries in turn, in the socioeconomic pathway between education and health.

\section{P1-207 ASSESSMENT OF PERINATAL HEALTH SYSTEM AT THE POOREST REGIONS IN BRAZIL TO DECREASE INEQUALITIES IN INFANT MORTALITY}

doi:10.1136/jech.2011.142976d.100

${ }^{1,2}$ S Lansky, ${ }^{*}$ I H Perpetuo, ${ }^{1} E$ França, ${ }^{3} S$ Bittencourt, ${ }^{3} \mathrm{~S}$ Granado, ${ }^{3} \mathrm{M}$ do $\mathrm{C}$ Leal. ${ }^{1}$ Belo Horizonte Health Department, Belo Horizonte, Minas Gerais, Brazil; ${ }^{2}$ Federal University of Minas Gerais, Belo Horizonte, Minas Gerais, Brazil; ${ }^{3}$ National School of Public Health-Fiocruzuz, Rio de Janeiro, Brazil

Since 2008 Brazil is implementing a Project to decrease infant mortality in the Northeast and the Amazon Regions and reduce inequalities in these poorest regions of the country. An analysis of perinatal health indicators for all the 17 states and capitals that comprise these regions was conducted in order to support health planning and management for an effective perinatal public health system. Perinatal healthcare and population's health indicators were arranged in six groups: socioeconomics and demographics, primary healthcare (coverage, access and quality of contraception and prenatal care), hospital birth care, organization of perinatal public health system (referral system, transport, specialised care and neonatal intensive care units), quality of information on deaths and mortality rates. A perinatal health score system was assembled based on the states' and capitals' achievement in 21 and 18 variables, respectively. Each variable received 1 to 3 points to classify states and capitals in three levels: poor $(<33.3 \%)$, intermediate $(33.3 \%$ to $66.6 \%)$ and satisfactory ( $\geq 66.7 \%)$. Ten $(58.8 \%)$ states and four $(23.5 \%)$ capitals were classified into the "poor" group. Only 1 state and 6 capitals achieved the "satisfactory" group. "Organization of the perinatal public health system" and "infant and early neonatal mortality rates" were the groups with the lowest results for states and capital, as well as the "primary healthcare" group for capitals. This score system revealed which states and capitals must be prioritised in specific health actions to improve perinatal health, prevent avoidable infant deaths and decrease inequalities in the country. 